\title{
Uma análise quantitativa da motivação e estratégia de estudo em física
}

\begin{abstract}
Alcides Goya
José Aloyseo Bzuneck

Ivo Aparecido Goulart

Resumo

O objetivo deste estudo foi avaliar o grau de motivação e estratégia pessoal de estudo de física dos alunos do ensino médio, do curso técnico e das engenharias, bem como a relação deste grau com as atividades práticas de laboratório. Os alunos foram avaliados por meio de questionários em escala Likert na orientação à meta de realização aprender física e estratégia pessoal de estudo de física. O questionário passou pelo teste KMO (Kaiser-Meyer-Olkin) e pelo teste de Bartlett de esfericidade e a aplicação da análise fatorial identificou os dois fatores correspondentes às variáveis estudadas. Os alunos do ensino médio apresentaram um aumento na motivação, mas não na estratégia. Os alunos do ensino técnico e das engenharias apresentaram um alto grau no início do curso e revelaram uma queda nas duas variáveis.
\end{abstract}

Palavras-chave: Motivação, meta de realização, estratégia pessoal de estudo, ensino de física.

\begin{abstract}
The aim of this study was to assess the degree of motivation and study's personal strategies use in physics by high school, technical education and engineering students as well as the relationship of this degree with the practical activities of the laboratory. Students were evaluated through Likert-tipe questionnaires on achievement goals as motivational construct and learning strategies use in the study of physics. The questionnaire was factor analyzed through KMO (Kaiser-Meyer-Olkin) test and by Bartlett's sphericity test. The high school students showed an increase in motivation, but not in the strategy. The technical education and engineering students showed a high level early in the course and showed a fall in both variables.
\end{abstract}

Key words: motivation, goal achievement, personal strategy for study, teaching of physics. 


\section{Introdução}

A motivação do aluno é considerada variável complexa e multifatorial, para cuja compreensão surgiu, no âmbito da Psicologia Educacional, diversas teorias e abordagens. Uma revisão feita alguns anos atrás identificou muitos sentidos ou construtos diferentes que se vinculam às diferentes linhas de pesquisas (MURPHY \& ALEXANDER, 2000). Apesar da complexidade do seu estudo, a influência das perspectivas motivacionais continua atraindo a atenção dos pesquisadores em ensino de ciências (BRITNER \& PAJARES, 2006; ABRAHAMS, 2009; SILVA et al, 2011; JOLY \& PRATES, 2011), pois o aluno motivado além de investir tempo, persiste nas atividades de aprendizagem, apesar das dificuldades (MAEHR \& MEYER, 1997; BZUNECK, 2009) e emprega estratégias eficazes (APPLETON et al., 2006).

Uma característica das tendências contemporâneas no estudo da motivação do aluno é a prevalência das abordagens sociocognitivistas. Segundo PINTRICH (2003), desenvolveram-se cinco famílias básicas de construtos sociocognitivos que se propõe a dar uma resposta sobre o que é que motiva os estudantes na sala de aula: crenças de auto-eficácia e de competência; atribuições e crenças de controle; interesse e motivação intrínseca; valorização; e metas diversas, especificamente as metas sociais e as metas de realização. Dentro dessa abordagem, o presente estudo se encontra especificamente na orientação à meta de realização aprender.

A orientação à meta de realização aprender denota um tipo específico de motivação na escola, que acentua os processos de aprender como objetivo pessoal do aluno (AMES, 1992; BZUNECK, 2009; GRANT \& DWECK, 2003). Das duas metas principais que foram contempladas nessa teoria - meta aprender e meta performance - a primeira é operacionalizada com itens que representam a orientação do aluno para dominar os conteúdos, para a compreensão e para desenvolver habilidades, sem preocupação com aparecer ou ser o melhor da classe. Ter a preocupação de aparecer, de ser o melhor caracteriza a meta performance, hoje subdividida em perfomance-aproximação e performance-evitação (BZUNECK, 2009). Em particular, um aluno direcionado à meta aprender, valoriza o aprender em si, mesmo que não sinta prazer nas atividades relacionadas ou interesse pessoal por elas. Ele responde bem a desafios, sem preocupação com aparecer. Envolvimento de qualidade, uso de estratégias de profundidade e emoções positivas apareceram associadas a essa modalidade motivacional. Nas mesmas pesquisas que comprovaram esses efeitos foram também identificados os fatores socioambientais responsáveis pela formação das metas de realização.

Se os processos motivacionais promovem engajamento nas atividades de aprendizagem, esse envolvimento consiste, mais especificamente, no emprego de estratégias de aprendizagem (APPLETON et al., 2006) que se balizam num outro referencial teórico conhecido como teoria de processamento de informação (BZUNECK, 2004; BORUCHOVITCH, 2005). Estratégias de aprendizagem são ações mentais e comportamentos com o objetivo de influenciar o processo de codificação e assim facilitar a aquisição e a recuperação das informações armazenadas na 
memória de longa duração (BORUCHOVITCH, 1999). Elas representam o saber como fazer, como estudar, como aprender. Isto explica o fato atestado amplamente na literatura de que estratégias eficazes de estudo resultam geralmente em melhor aprendizagem. Entre as taxonomias de estratégias, a de PINTRICH (1989) consiste no agrupamento em três grandes categorias, que ele denominou de estratégias cognitivas (como a de elaboração e a de organização), metacognitivas (planejamento, monitoramento) e de gerenciamento de recursos, que incluem o tempo e o ambiente estudo.

A estratégia de elaboração auxilia os alunos a armazenarem a informação na memória de longa duração por meio de conexões que se estabelecem entre a nova informação e os conhecimentos prévios, proporcionando uma melhor armazenagem e consequente recuperação da informação. As estratégias de organização referem-se à atividade do aprendiz para identificar as idéias principais do novo conteúdo e estabelecer ligações entre as partes, sendo que a organização também dependerá dos conhecimentos prévios que o sujeito possa ativar. Parece confirmar o que dizia Ausubel há quase cinquenta anos atrás: "conhecimento prévio é o fator isolado que mais influencia na aprendizagem“ (Ausubel apud MOREIRA \& GRECA, 2003, p.302).

As estratégias metacognitivas são definidas como cognições sobre cognições, o aprendiz ao planejar as atividades reflete sobre a própria aprendizagem estabelecendo metas de estudo e análise das tarefas. Monitora checando sua compreensão, definindo a necessidade ou não de uma releitura, bem como buscando uma melhor compreensão do conteúdo lido. Por fim as estratégias de gerenciamento de recursos dizem respeito ao controle do tempo, do apoio externo, do ambiente físico de estudo e até do esforço e persistência (PINTRICH, 1999).

Apesar da complexidade dessas perspectivas motivacionais no ensino de ciências, dentro dos referenciais teóricos comentados acima, pode se dizer que estas estratégias de aprendizagem possivelmente serão adotadas de acordo com as metas de realização assumidas pelo aprendiz (PINTRICH, 1999; SANTOS, 2006). Por outro lado, sabe-se que os problemas na motivação do aluno variam e podem se modificar em função do avanço nas séries escolares. Essas variações na motivação ocorrem tanto em função do nível e série escolar quanto em função do tipo de disciplina (STIPEK, 1998). Por exemplo, a motivação de alunos de cursos superiores já é o resultado de todas as experiências anteriores com professores e contextos e a maioria deles já desenvolveu uma autopercepção de capacidade e crenças num grau que pode favorecer ou prejudicar a motivação. Além disso, há o componente da motivação, que consiste na opção vocacional ou definição por uma meta de vida.

Portanto, o objetivo deste trabalho foi fazer um estudo quantitativo da motivação à meta de realização aprender física e estratégia pessoal de estudo de física através da aplicação de um mesmo questionário aos alunos do primeiro ano de três cursos: ensino médio, curso técnico e engenharia. Foi feito um estudo comparativo do grau de motivação e estratégia entre o 
início e fim de um ano no ensino médio e no curso técnico e, também, entre o início e o fim de um semestre nas engenharias em função da utilização de algumas atividades experimentais.

Nos três cursos foram feitos experimentos (movimento uniforme, movimento uniformemente variado e lançamentos de projéteis) utilizando os trilhos idealizados pelo primeiro autor, fato que não ocorreu nos respectivos grupos de controle. 2 AMOSTRA, MÉTODO E PROCEDIMENTOS A pesquisa foi feita com 316 alunos do primeiro ano, sendo 86 alunos do ensino médio de uma escola da rede pública e 230 alunos de uma instituição federal, sendo 121 do curso técnico - eletrotécnica e mecânica - e 109 das engenharias: elétrica e mecânica. A amostra total corresponde a 491 questionários respondidos, uma vez que 175 responderam duas vezes, no início e no final da disciplina, e os restantes 141 responderam apenas uma vez, no final da disciplina. Neste trabalho não serão feitas distinções entre cursos específicos - eletrotécnica e mecânica para o técnico ou entre elétrica e mecânica para as engenharias-, pois as análises estatísticas da nossa amostra indicam que não houve diferenças significativas entre eles nas duas variáveis estudadas, tanto no ensino técnico como nas engenharias.

A parte principal da amostra, o grupo experimental, corresponde aos 175 alunos que repetiram os testes, 47 pertenciam ao primeiro ano do ensino médio, 62 pertenciam ao primeiro ano da escola técnica e 66 ao primeiro semestre das engenharias, ou seja, todos eram "calouros" dos seus respectivos cursos. O terceiro autor deste artigo foi o professor do primeiro ano da escola técnica, o primeiro autor foi o professor dos calouros das engenharias e três alunos estagiários das engenharias fizeram os experimentos para o professor do ensino médio. No ensino médio, os estagiários fizeram experimentos de movimento uniforme, movimento uniformemente variado e lançamentos de projéteis utilizando os trilhos idealizados pelo primeiro autor. Tanto no curso técnico como nas engenharias foram ministradas duas aulas de laboratório por semana e, entre os diversos experimentos, constavam os mesmos que foram aplicados no ensino médio com o uso dos trilhos. A segunda parte da nossa amostra, o grupo de controle, foi composta por 141 alunos, todos também do primeiro ano e pertencentes às mesmas instituições de ensino do grupo experimental: 39 alunos do ensino médio da mesma escola pública, 59 do ensino técnico e 43alunos das engenharias da mesma instituição federal. Esses alunos responderam apenas uma vez o questionário. Portanto, as diferenças entre o grupo experimental e o grupo de controle variaram em função dos cursos. Nos três cursos foram feitos experimentos (movimento uniforme, movimento uniformemente variado e lançamentos de projéteis) utilizando os trilhos idealizados pelo primeiro autor, fato que não ocorreu nos respectivos grupos de controle. Porém, enquanto esses experimentos foram os únicos que os alunos do ensino médio realizaram, os alunos do ensino técnico e das engenharias realizaram outros experimentos, sem o trilho, ao longo do ano e do semestre, respectivamente.

Os 316 alunos responderam ao mesmo questionário que era composto de 20 questões apresentadas na escala Likert, 13 questões estavam relacionadas à motivação meta de 
realização aprender física e sete questões relacionadas à estratégia pessoal de estudo de física. O questionário passou pelo teste KMO (Kaiser-Meyer-Olkin) para verificar a aplicação da análise fatorial. O resultado expresso neste teste $(0,907)$ demonstra um excelente grau de ajuste para aplicação da técnica multivariada análise fatorial, sendo ratificada pelo teste de Bartlett de esfericidade ao nível de significância 0,000. Na análise fatorial, ao verificar as cargas fatoriais na extração dos componentes principais, foram eliminadas algumas questões e o questionário original ficou reduzido a 13 questões, tal como é apresentado no apêndice 1.

A análise fatorial no questionário reduzido indicou que $47,29 \%$ das cargas fatoriais correspondiam a dois fatores. O mesmo número de fatores foi obtido ao se fazer a análise dos autovalores maiores que 1,0 . Os fatores extraídos através da rotação Varimax foram submetidos ao teste de confiabilidade alfa de Cronbach gerando os seguintes valores: meta de realização aprender (alfa $=0,85$ ) e estratégia pessoal de estudo (alfa $=0,72$ ). Esses resultados indicam que o construto e as escalas utilizadas apresentam uma boa confiabilidade interna para uma pesquisa xploratória. O primeiro fator, escala de orientação à meta de realização aprender física (MRF), ficou composto por oito perguntas (apêndice um). Cada pergunta apresenta como resposta cinco itens a serem marcados numa escala tipo Likert de 1 (nada verdadeiro) até 5 (totalmente verdadeiro). Exemplo: " Para os estudos de Física eu aproveito bem o tempo que tenho fora das aulas". Através dessas simples perguntas, esta escala procura medir o grau de motivação para dominar os conteúdos, para a compreensão e para desenvolver habilidades.

O segundo fator, escala de estratégia pessoal de estudo de física (EPE), é composto por cinco perguntas (apêndice 1). Como na escala anterior, as perguntas apresentam como resposta cinco itens a serem marcados numa escala tipo Likert de 1 (nada verdadeiro) até 5 (totalmente verdadeiro). Exemplo: "Venho para as aulas de física sem ter lido nada sobre a matéria a ser dada". Apesar da limitação deste questionário, através desta escala procura-se medir, em certo aspecto, o grau de estratégias cognitivas e de gerenciamento de recursos em relação à aprendizagem de física.

\section{Resultados e comentários}

A maior parte dos resultados deste trabalho é apresentada em tabelas comparativas dos escores médios grupais nas duas variáveis: MRF (orientação à meta de realização aprender física) e EPE (estratégia pessoal de estudo de física).

A tabela 1 é uma apresentação geral das amostras coletadas nas duas variáveis MRF e EPE. Os números nesta primeira tabela são apresentados apenas com os cálculos das médias para cada turma, sem nenhum tratamento estatístico. Portanto, os pontos assinalados abaixo servem apenas como orientações para as análises estatísticas que serão feitas em seguida. As turmas do ensino médio começam com a letra M: Ma corresponde ao início do primeiro ano, 
$\mathrm{Mb}$ ao término do primeiro ano e Mc corresponde ao grupo de controle. As turmas do curso técnico começam com a letra $\mathrm{T}$ : Ta corresponde ao início do primeiro ano, Tb ao término do primeiro ano e Tc para o grupo de controle. Os calouros das engenharias começam com a letra E: Ea no início do semestre, Eb no fim do semestre e Ec para o grupo de controle.

Tabela 1: Grau de orientação à meta de realização aprender física (MRF) e estratégia pessoal de estudo de física (EPE) de todas as turmas.

\begin{tabular}{|c|c|c|c|c|c|}
\hline Turma & $\mathrm{N}$ & $\mathrm{MRF}$ & $\mathrm{DP}$ & $\mathrm{EPE}$ & $\mathrm{DP}$ \\
\hline $\mathrm{Ma}$ & 47 & 2,14 & 0,81 & 3,13 & 0,91 \\
\hline $\mathrm{Mb}$ & 47 & 2,57 & 0,87 & 3,14 & 0,92 \\
\hline $\mathrm{Mc}$ & 39 & 2,32 & 0,68 & 2,82 & 1,02 \\
\hline $\mathrm{Ta}$ & 62 & 3,56 & 0,60 & 4,08 & 0,60 \\
\hline $\mathrm{Tb}$ & 62 & 3,20 & 0,74 & 3,83 & 0,77 \\
\hline $\mathrm{Tc}$ & 59 & 2,85 & 0,78 & 3,37 & 0,73 \\
\hline $\mathrm{Ea}$ & 66 & 3,55 & 0,63 & 4,07 & 0,55 \\
\hline $\mathrm{Eb}$ & 66 & 3,20 & 0,68 & 3,75 & 0,70 \\
\hline $\mathrm{Ec}$ & 43 & 2,79 & 0,77 & 3,40 & 0,71 \\
\hline
\end{tabular}

Ma-início do 1o ano do e. médio $\mathrm{Mb}$ - final do 1o ano do e. médio

Mc-grupo de controle do e. médio Ta-início do 1o ano do técnico

Tb-final do 1 o ano do técnico Tc-grupo de controle do técnico

Ea-início do 1 o sem das engenharias Eb-final do $1^{\circ}$ sem das engenharias

Ec-grupo de controle das engenharias

Em síntese, a Tabela 1 revela os seguintes dados:

a) Em todas as turmas o grau de MRF é menor do que o grau de EPE, ou seja, a orientação à meta de realização aprender física é menor do que a estratégia pessoal de estudo. Inclusive a média de $\operatorname{MRF}(2,91)$, calculada entre as nove turmas, está abaixo do ponto médio $(3,00)$ enquanto a média de $\operatorname{EPE}(3,51)$, também calculada entre as nove turmas, está bem acima, tendo em conta que o valor máximo é cinco. Os alunos do ensino médio e dos grupos de controle estão abaixo das médias da amostra total, nas duas variáveis.

b) Os calouros no início do curso, tanto do técnico ( $\mathrm{Ta}$ ) como das engenharias (Ea) aparecem com os maiores índices, tanto na variável MRF $(3,56$ e 3,55) como na variável EPE $(4,08$ e 4,07). Além dessa forte coincidência nos valores é digno de nota o alto índice atingido na 
variável EPE, i.e., na média, essas duas turmas responderam ao questionário do apêndice 1, dando uma resposta um pouco acima do "bastante verdadeiro".

c) Ao compararmos as mesmas turmas, entre o início e o final, no ensino médio houve um aumento de grau e nos outros dois cursos houve declínio.

A variação nas duas escalas, comentada no último ponto (c), foi analisada separadamente, primeiro entre os alunos do ensino médio, depois entre os alunos do curso técnico e finalmente entre os alunos das engenharias, tanto pela anova (conforme as tabelas 2, 3 e 4) como pelo teste de Tukey.

A tabela 2 apresenta os dados da análise de variância anova aplicada às médias nas duas variáveis, entre os alunos do ensino médio: $\operatorname{MRF}(F=3,56$ e $p=0,03)$ e EPE ( $F=1,51$ e $p$ $=0,22)$. A variabilidade entre os grupos é, cerca de 3,6 vezes para a variável MRF e 1,5 vezes para EPE, maior do que dentro dos grupos, mas o $p$-valor é menor que o nível de significância $\alpha=0,05$ apenas para a variável MRF.

Tabela 2: Anova relativo aos alunos do ensino médio

\begin{tabular}{|c|c|c|c|c|c|c|c|c|}
\hline & \multicolumn{2}{|c|}{ Ma (N=47) } & \multicolumn{2}{c|}{ Mb (N=47) } & \multicolumn{2}{c|}{ Mc $(\mathrm{N}=39)$} & $\mathrm{F}$ & $\mathrm{p}$ \\
\hline & Média & DP & Média & DP & Média & DP & & \\
\hline MRF & 2,14 & 0,81 & 2,57 & 0,87 & 2,32 & 0,68 & 3,56 & 0,03 \\
\hline EPE & 3,13 & 0,91 & 3,14 & 0,92 & 2,82 & 1,02 & 1,51 & 0,22 \\
\hline
\end{tabular}

$\mathrm{Ma}$ - início do $1^{\circ}$ ano do médio $\mathrm{Mb}$ - final do $1^{\circ}$ ano do médio

$\mathrm{Mc}$ - grupo de controle do médio

O teste de Tukey especificou que, na variável MRF, há uma diferença significativa apenas entre $M a$ e $M b(p=0,02)$, pois as outras diferenças não são significativas nem entre $M a$ e $M c(p=0,54)$ e nem entre $M b$ e $M c(p=0,31)$. Esse resultado mostra que o grupo experimental se destacou na variável motivacional, ou seja, a atividade prática com os trilhos fez com que, na média, os alunos tivessem uma maior motivação, contudo não melhorassem nas estratégias pessoais de estudo.

A tabela 3 apresenta os dados da análise de variância anova aplicada às médias nas duas variáveis, entre os alunos do curso técnico: $\operatorname{MRF}(F=15,51$ e $p \leq 0,00)$ e $\operatorname{EPE}(F=15,81$ e $p \leq 0,00)$. A variabilidade entre os grupos é cerca de quinze vezes maior do que dentro dos grupos e o $p$ valor é bem menor que o nível de significância $\alpha=0,05$.

Tabela 3: Anova relativo aos alunos do ensino técnico

\begin{tabular}{|c|c|c|c|c|c|c|c|c|}
\hline & \multicolumn{2}{|c|}{$\mathrm{Ta}(\mathrm{N}=62)$} & \multicolumn{2}{|c|}{$\mathrm{Tb}(\mathrm{N}=62)$} & \multicolumn{2}{|c|}{$\mathrm{Tc}(\mathrm{N}=59)$} & \multirow[t]{2}{*}{ F } & \multirow[t]{2}{*}{$\mathrm{p}$} \\
\hline & Média & DP & Média & DP & Média & DP & & \\
\hline MRF & 3,56 & 0,60 & 3,20 & 0,74 & 2,85 & 0,78 & 15,51 & 0,000 \\
\hline EPE & 4,08 & 0,60 & 3,83 & 0,77 & 3,37 & 0,73 & 15,81 & 0,000 \\
\hline
\end{tabular}

$\mathrm{Ta}$ - início do $1^{\circ}$ ano do técnico $\mathrm{Tb}$ - final do $1^{\circ}$ ano do técnico

$\mathrm{Tc}$ - grupo de controle do técnico 
O teste de Tukey especificou que as diferenças na orientação à meta de realização aprender física MRF são significativos em todas as comparações: Ta com Tb $(p=0,01)$, Ta com Tc $(p \leq 0,00)$ e entre Tb e Tc $(p=0,00)$. Algo diferente ocorre com o teste de Tukey quando aplicado na variável estratégia pessoal de estudo EPE: Ta com Tb $(p=0,11)$, Ta com Tc $(p \leq 0,00)$ e entre Tb e Tc $(p=0,02)$. Isso indica que o aluno recém-ingressado na escola técnica, depois de ser aprovado num teste seletivo, possui um grau alto de motivação e estratégia de estudo, mas ao longo do primeiro ano, esse alto grau decai significativamente em relação à orientação à meta de realização aprender física e não significativamente em relação à estratégia pessoal de estudo de física. Mesmo com essa queda, quando comparados com o grupo de controle, os alunos do grupo experimental chegaram ao fim do ano com graus significativamente superiores nas duas variáveis.

Tabela 4: Anova relativo aos alunos das engenharias

\begin{tabular}{|l|c|c|c|c|c|c|c|c|}
\hline & \multicolumn{2}{|c|}{ Ea $(\mathrm{N}=66)$} & \multicolumn{2}{c|}{ Eb $(\mathrm{N}=66)$} & \multicolumn{2}{c|}{ Ec $(\mathrm{N}=43)$} & $\mathrm{F}$ & $\mathrm{p}$ \\
\hline & Média & DP & Média & DP & Média & DP & & \\
\hline MRF & 3,55 & 0,63 & 3,20 & 0,68 & 2,75 & 0,74 & 18,15 & 0,000 \\
\hline EPE & 4,07 & 0,55 & 3,75 & 0,70 & 3,42 & 0,69 & 13,43 & 0,000 \\
\hline
\end{tabular}

$\mathrm{Ea}$ - início do $1^{\circ}$ sem da engenharia $\quad \mathrm{Eb}$ - final do $1^{\circ}$ sem da engenharia

$\mathrm{Ec}$ - grupo de controle da engenharia

O declínio nas duas variáveis, MRF e EPE, entre os alunos das engenharias, também foi analisado pelo tratamento estatístico anova conforme mostra a tabela 4. Ela apresenta os dados da análise de variância anova aplicada às médias nas duas variáveis: $\operatorname{MRF}(F=18,15$ e $p \leq 0,00)$ e $\operatorname{EPE}(F=13,43$ e $p \leq 0,00)$. A variabilidade entre os grupos ficou entre 13 a 18 vezes maior do que dentro dos grupos e o $p$-valor foi menor que o nível de significância $\alpha=0,05$.

O teste de Tukey especificou que as diferenças na orientação à meta de realização aprender física MRF são significativos em todas as comparações: Ea com $E b(p=0,00)$, Ea com Ec $(p \leq 0,00)$ e entre $E b$ e $E c(p=0,00)$. O mesmo ocorreu com o teste de Tukey quando aplicado na variável estratégia pessoal de estudo EPE: $\operatorname{Ea} c o m \operatorname{Eb}(p=0,01)$, Ea com $E c(p \leq 0,00)$ e entre Eb e $E c(p=0,03)$. Isso indica que os alunos recém-ingressados nas engenharias, depois de serem aprovados nos vestibulares, chegam com um grau alto de orientação à meta de realização aprender e estratégia pessoal de estudo de física, mas acabam perdendo significativamente até o final do semestre. No entanto, mesmo assim, o grau nessas duas variáveis ainda é significativamente superior quando comparado com o grupo de controle.

Para finalizar as comparações, a amostra total apresentada na tabela 1 é dividida de acordo com os três cursos: 1 o ano do ensino médio $M(N=71), 10$ ano do curso técnico $T$

$(\mathrm{N}=183)$ e 1 o ano das engenharias.

Tabela 5: Anova relativo aos alunos do ensino médio, técnico e engenharias. 


\begin{tabular}{|l|c|c|c|c|c|c|c|c|}
\hline & \multicolumn{2}{|c|}{$\mathrm{M}(\mathrm{N}=133)$} & \multicolumn{2}{c|}{$\mathrm{T}(\mathrm{N}=183)$} & \multicolumn{2}{c|}{$\mathrm{E}(\mathrm{N}=175)$} & $\mathrm{F}$ & $\mathrm{p}$ \\
\hline & Média & DP & Média & DP & Média & DP & & \\
\hline MRF & 2,34 & 0,82 & 3,21 & 0,76 & 3,22 & 0,74 & 61,46 & 0,000 \\
\hline EPE & 3,04 & 0,95 & 3,77 & 0,76 & 3,79 & 0,71 & 41,70 & 0,000 \\
\hline
\end{tabular}

$\mathrm{M}-1^{\circ}$ ano do ensino médio

$\mathrm{T}-1^{\circ}$ ano do curso técnico

$\mathrm{E}-1^{\circ}$ ano das engenharias

A análise de variância anova, apresentada na tabela 5 , mostra que há diferenças entre as médias: $\operatorname{MRF}(F=61,46$ e $p \leq 0,00)$ e EPE $(F=41,70$ e $p \leq 0,00)$. A variabilidade entre os grupos fica entre 41,7 a 61,5 vezes maiores do que dentro dos grupos e o $p$-valor é bem menor que o nível de significância $\alpha=0,05$.

O teste de Tukey especificou que as diferenças na orientação à meta de realização aprender física MRF são significativas em duas comparações: $M$ com $T(p \leq 0,00)$ e $M$ com $E$ $(p \leq 0,00)$. Mas há uma igualdade também significativa entre $T$ e $E(p \geq 0,99)$. Algo similar ocorreu com o teste de Tukey quando aplicado na variável estratégia pessoal de estudo EPE:

$M \operatorname{com} T(p \leq 0,00), M$ com $E(p \leq 0,00)$ e entre $T$ e $E(p=0,95)$.

Portanto, os alunos do 10 ano do ensino médio estão com grau inferior ao ponto médio na orientação à meta de realização aprender física MRF e praticamente no ponto médio na estratégia pessoal de estudo de física. E quando comparados com os alunos do 10 ano do ensino técnico e com os 1으 ano das engenharias, apresentam um grau significativamente menor. Já os alunos do ensino técnico e das engenharias apresentam um grau significativamente igual nas duas variáveis.

Para finalizar o tratamento estatístico, foram calculadas as correlações entre as variáveis MRF e EPE para a amostra total $(N=491, r=0,57, p=0,00)$. Mesmo não se referindo a um mesmo construto, as duas medidas compartilham algumas características, i.e., alunos que apresentam alto grau de motivação também possuem alto grau na estratégia pessoal de estudo, realidade fácil de conferir pelas médias da tabela 1.

\section{Considerações finais}

Devido à complexidade do construto, uma vez que a motivação do aluno é uma variável multifatorial, neste trabalho nos restringimos a estudar o grau de orientação à meta de realização aprender e o grau de estratégia pessoal de estudo. Um resumo dos principais resultados obtidos foi os três aspectos destacados a partir dos dados da tabela 1 , frutos de um tratamento quantitativo e confirmado pela análise estatística anova e pelo teste de Tukey, conforme os dados apresentados nas tabelas 2 a 5 .

Esses dados não somente mostraram que o grau de meta de realização ficou menor do que o grau de estratégia pessoal em todas as turmas analisadas, mas mostraram também que a própria variação na motivação é maior do que a variação na estratégia, fato observado nos três 
cursos. A evolução do grau de motivação e estratégia em função da utilização de algumas atividades experimentais ficou mais clara nos resultados apresentados pelos alunos do ensino médio, uma vez que eles não tiveram outras atividades experimentais. Os dados mostraram que houve um aumento na orientação à meta de realização aprender física, ou seja, as atividades experimentais realizadas com o uso dos trilhos influenciaram positivamente na motivação dos alunos para a aprendizagem, confirmando quantitativamente a análise qualitativa que o professor daquela escola pública tinha feito e comunicado ao primeiro autor.

Esse fato incentiva o uso dos trilhos em outras turmas e em outras escolas públicas, pois é sabido que as atividades práticas influenciam positivamente nas motivações dos alunos (LAVONE, 2004; LABURÚ et al., 2011).

Entretanto, aparentemente os efeitos dessas mesmas atividades experimentais no curso técnico e nas engenharias ficaram ocultos, pois foi observada uma queda nas duas variáveis.

Existem muitas razões que poderiam ser levantadas para explicar essa queda, muitas provavelmente estejam fora do âmbito desta pesquisa. Porém, não se pode deixar de considerar que as atividades experimentais realizadas com o uso dos trilhos ficaram proporcionalmente em menor número quando comparadas com outras atividades práticas, tanto no curso técnico como nas engenharias. Ou seja, as atividades experimentais com os trilhos provavelmente possuiam mais carga de novidade para os alunos do ensino médio do que para os alunos do ensino técnico e das engenharias, pois estes últimos já estavam mais acostumados com as aulas práticas. Outro fator importante foi a disparidade no grau de motivação e estratégia no início do curso: baixo no ensino médio e alto no curso técnico e nas engenharias.

Por outro lado, é importante ressaltar que, apesar da queda, o nível de motivação e estratégia continuou não somente acima do ponto médio como significativamente superior ao nível do grupo de controle. Além disso, esses alunos do grupo experimental, tanto do curso técnico como das engenharias, chegaram mais motivados no fim do semestre e com grau maior em estratégias quando comparados com os melhores níveis alcançados pelos alunos do ensino médio. Como explicação plausível a esse dado específico, pode-se atribuir essa disposição positiva à opção vocacional pelo respectivo curso superior e à carreira profissional futura, aliada à consciência de que toda disciplina do curso confere para suas metas de vida. A literatura (OETTINGEN e MAYER, 2002; SIMONS et al., 2004) tem comprovado consistentemente o efeito motivacional de metas de vida sobre o envolvimento nos estudos, ao menos quando se trata de alunos que, de modo pensado, já se definiram por uma carreira.

Além disso, podemos destacar o alto grau de motivações que muitos alunos possuem quando chegam às universidades federais, especialmente logo depois de superarem muitos concorrentes no curso escolhido. Ao mesmo tempo, no decorrer do semestre, principalmente num curso de engenharia, é natural que os calouros encontrem dificuldades, sendo difícil 
manter o mesmo grau de motivação e, consequentemente de estratégias, pois uma está relacionada com outra (PINTRICH, 1999; SANTOS, 2006).

Como última consideração, destaca-se a similaridade observada entre os alunos do curso técnico e os da engenharia, nas duas variáveis, pois a igualdade foi medida tanto quando se comparou os alunos do 1 o ano do curso técnico com os calouros das engenharias, como quando se comparou os respectivos grupos de controle. Se por um lado o curso técnico é distinto das engenharias, pois os professores são diferentes, as idades e as origens dos alunos são diferentes, etc.; por outro lado há muitas semelhanças, pois eles passaram por um teste de seleção equivalente, em certos aspectos fizeram opções por carreiras semelhantes, estudam na mesma instituição federal de ensino, etc.

Enfim, o questionário possibilitou avaliar o grau de motivação e estratégia pessoal de estudo de física dos alunos do ensino médio, do curso técnico e das engenharias, bem como verificar a relação deste grau com a utilização dos trilhos no ensino médio. Acredita-se que os resultados apresentados abrem perspectivas para que os trilhos possam ser utilizados especialmente no ensino médio da rede pública. Uma possível validação do questionário poderia auxiliar o (a) professor (a), tanto do ensino médio como universitário, a medir o grau de motivação à meta de realização aprender física e estratégia pessoal de estudo em física dos seus alunos. 


\section{Referência}

ABRAHAMS, I. Does Practical Work Really Motivate? A study of the affective value of practical work in secondary school science. International Journal of Science Education, v. 31, n. 17, p. 2335-2353, 2009.

AMES, C.. Classrooms: Goals, structures, and student motivation. Journal of Education Psychology, v. 84, p. 261-271, 1992.

APPLETON, J.J.; CHRISTENSON, S.L.; KIM, D. e RESCHLY, A. L.. Measuring cognitive and psychological engagement: Validation of the Student Engagement Instrument. Journal of School Psychology, 44, p. 427-445, 2006.

BORUCHOVITCH, E.. Estratégias de aprendizagem: Contribuições para a formação de professores nos cursos superiores. In: Joly, M. C. R. A., Santos, A. A. A. dos e Sisto, F. F. (orgs.). Questões do Cotidiano Universitário. São Paulo: Casa do Psicólogo, p. 239-260, 2005.

BORUCHOVITCH, E.. Estratégias de aprendizagem e desempenho escolar: Considerações para a prática educacional. Psicologia: Reflexão e Crítica, 12, n. 2, p. 361-367, 1999.

BRITNER S. L.; PAJARES, F.. Sources of Science Self-Efficacy Beliefs of Middle School Students. Journal of Research in Science Teaching, v. 43, n. 5, p. 485-499, 2006.

BZUNECK, J. A.. A Motivação do Aluno: Aspectos Introdutórios. Em Boruchovitch, E. e Bzuneck, J. A (orgs). A Motivação do Aluno: Contribuições da Psicologia Contemporânea (p. 9-36). Petrópolis, ed. Vozes. 2009.

BZUNECK, J.A. Aprendizagem por processamento da informação: uma visão construtivista. In: Boruchovitch E. e Bzuneck J. A. (orgs). Aprendizagem: processos psicológicos e o contexto social na escola. Petrópolis, ed.Vozes, p.17-54, 2004.

GRANT, H. \& DWECK, C. S.. Clarifying achievement goals and their impact. Journal of Personality and Social Psychology, v. 85, n. 3, p. 541-553, 2003.

JOLY, M. C. R. A. \& PRATES, E. A. R. . Avaliação da Escala de Motivação Acadêmica em estudantes paulistas: propriedades. Psico-USF. Itatiba, 16, n. 2, p. 175-184, 2011.

LABURÚ, C. A., MAMPRIN, M.I. de L. L e SALVADEGO, W. N. C., Professor das ciências naturais e a prática de atividades experimentais no ensino médio -uma análise segundo Charlot.. Londrina: Eduel, 2011.

LAVONEN, J., JAUHIAINEN, J., KAPONEN, J. e KURKI-SUONIO, K. Effect of a long-term in-service training program on teacher's beliefs about the role of experiments in physics education. International Journal of Science Education, Londres, v. 26, n. 3, p. 309-328, 2004. 
MAEHR, M. L. \& MEYER, H. A. . Understanding Motivation and Schooling: Where We've Been, Where We Are, and Where We Need to Go. Educational Psychology Review, v. 9, n.4, p. 371-409, 1997.

MOREIRA, M. A. \& GRECA, I. M.. Mudança conceitual: análise crítica e propostas à luz da teoria da aprendizagem significativa. Ciência e Educação, Bauru, v. 9, n. 2, p. 301-315, 2003.

MURPHY, P. K. \& ALEXANDER, P. A. A motivated exploration of motivation terminology. Contemporary Educational Psychology, v. 25, p. 3-5, 2000.

OETINGEN, G. \& MAYER, D. The motivating function of thinking about the future: Expectations versus fantasies. Journal of Personality and Social Psychology, v. 83, p. 1198-1212, 2002.

PINTRICH, P. R. A motivational science perspective on the role of student motivation in learning and teaching contexts. Journal of Educational Psychology, v. 95, p. 667-686, 2003.

PINTRICH, P. R.. The dynamic interplay of student motivation and cognition in the college classroom. In C. Ames, \& M. Maehr, Advances in motivation and achievement: Motivation enhancing environments, Greenwich, CT: JAI Press, 1989, vol. 6, p. 117-160.

PINTRICH, P.R.. The role of motivation in promoting and sustaining self-regulated learning. International Journal of Educational Research, v. 31, p. 459-470, 1999.

SANTOS, T. S. Percepções das avaliações e sua relação com motivação e estratégias de aprendizagem. Londrina, 112 p., 2006. Dissertação (Mestrado) - Universidade Estadual de Londrina.

SILVA, F. R.; BARROS, M. A.; LABURÚ, C. E. e DOS SANTOS, L. C. A. . Crenças de Eficácia, Motivação e a Fomação de Professores de Física. Caderno Brasileiro de Ensino de Física. Florianópolis, v. 28, n. 1, p. 214-228, 2011.

SIMONS, J.; VANSTEENKISTE, M.; LENS, W.; LACANTE, M., Placing Motivational and future time perspective theory in a temporal perspective. Educational Psychology Review, v.16, p. 121-139, 2004.

STIPEK, D. J. Motivation to learn: from theory to practice. Englewood Cliffs, NJ, Prentice Hall, 1998. 
Alcides Goya - Universidade Tecnológica Federal do Paraná Avenida dos Pioneiros, 3131 Londrina -PR - goya@utfpr.edu.br

José Aloyseo Bzuneck - Universidade Estadual de Londrina Rodovia Celso Garcia Cid - PR 445 Km380 Londrina-PR - bzuneck@sercomtel.com.br

Ivo Aparecido Goulart - ivag@utfpr.edu.br Universidade Tecnológica Federal do Paraná Avenida Alberto Carazzai, 1640 Cornélio Procópio- PR 